\title{
Predictors of phrenic nerve injury during pulmonary vein isolation for curing atrial fibrillation with balloon-based visually guided laser ablation.
}

\author{
Shinichi Tachibana ${ }^{1}$, Kaoru Okishige ${ }^{1}$, Kouzi Sudo ${ }^{1}$, Takatoshi Shigeta ${ }^{1}$, Yuichiro Sagawa ${ }^{1}$, \\ Rena Nakamura ${ }^{1}$, Manabu Kurabayashi ${ }^{1}$, Yasuteru Yamauchi ${ }^{1}$, Masahiko Goya ${ }^{2}$, and \\ Tetsuo Sasano ${ }^{2}$ \\ ${ }^{1}$ Yokohama City Minato Red Cross Hospital \\ ${ }^{2}$ Tokyo Medical and Dental University
}

July 16, 2020

\begin{abstract}
Introduction: Pulmonary vein (PV) isolation (PVI) with a balloon-based visually guided laser ablation (VGLA) is a useful tool for treating atrial fibrillation (AF), however, phrenic nerve injury (PNI) is an important complication. We investigated the predictors of developing PNI during VGLA. Methods and Results: This study included 130 consecutive patients who underwent an initial VGLA of non-valvular paroxysmal AF. During the ablation of the right-sided pulmonary veins, continuous and stable right phrenic nerve pacing was performed, and the compound motor action potentials (CMAPs) were recorded. Twenty patients developed PNI during the PVI. The patients who suffered from PNI had a significantly larger right superior PV (RSPV) ostium area $(284.7 \pm 47.0 \mathrm{~mm} 2$ vs. $233.1 \pm 46.4 \mathrm{~mm} 2, \mathrm{P}<0.01)$ than that of the other patients. Receiver operating characteristic analyses revealed that the area under the curve of the RSPV ostial area was 0.79 (95\% confidence interval: 0.69-0.90) with an optimal cut-off point of $238.0 \mathrm{~mm} 2$ (sensitivity: 0.58, specificity: 0.95). In the multivariate analyses, large RSPV ostial area (HR 1.02, 95\% confidence interval: 1.01-1.03, $\mathrm{P}<0.01$ ) and small balloon size (HR 0.72, 95\% confidence interval: 0.53-0.98, $\mathrm{P}=0.03)$ were independent risk factors for PNI. PNI remained in 13 patients after the procedure, but 12 of those patients recovered from PNI during the follow-up period. Conclusion: The incidence of PNI during the VGLA was relatively high, but the PNI improved in the majority of cases. During the VGLA, a large RSPV and small balloon size were predictors of PNI.
\end{abstract}

\section{Introduction}

Pulmonary vein isolation is an effective treatment of atrial fibrillation. Pulmonary vein isolation (PVI) with a balloon-based visually guided laser ablation (VGLA) is regarded as a useful tool for treating atrial fibrillation $(\mathrm{AF})_{1)}$. In a previous multicenter study, VGLA exhibited non-inferiority to radiofrequency ablation in terms of the efficacy and safety in treating paroxysmal and persistent $\mathrm{AF}_{2,3)}$. However, phrenic nerve injury (PNI) has been one of the important complications. A prior randomized controlled study of the PVI using either the VGLA or radiofrequency ablation showed that diaphragm paralysis occurred in $3.5 \%$ of the patients who underwent VGLA, which was higher than that of radiofrequency ablation ${ }_{2)}$. Although the incidence of PNI did not significantly differ between the ablation procedures using the cryoballoon (CB) and laser balloon (LB), PNI was a common perioperative complication $_{4}$. Balloon dilation causes right superior pulmonary vein (RSPV) strain and reduces the anatomical distance between the balloon and right phrenic nerve, which may lead to $\mathrm{PNI}_{5}$ ). In $\mathrm{CB}$ cases, a large RSPV maximal diameter and more distal positioning of the CB 
relative to the cardiac shadow are independent determinants for predicting $\mathrm{PNI}_{6}$ ). On the other hand, no predictors of PNI have been reported in VGLA cases. Nagase et al. reported that the compound motor action potentials (CMAPs) quickly declined during VGLA of the right superior pulmonary vein when PNI was provoked 7 . They concluded that it was unclear if the CMAP monitoring could prevent PNI during the VGLA. In this study, we investigated the predictors of PNI during the VGLA.

\section{Methods}

\section{Study population}

The subjects consisted of consecutive patients undergoing the VGLA as an initial procedure for paroxysmal AF (PAF) from July 2018 to January 2020. The subjects were consecutive patients undergoing AF ablation for standard clinical indications 1 . Written informed consent for the ablation procedures were obtained from all patients. The definition of PAF was according to the American Heart Association/American College of Cardiology/European Society of Cardiology guidelines, which is as follows: PAF was defined as AF that selfterminated within 7 days $_{1)}$. The study protocol was approved by the hospital's institutional human ethics committee. The study complied with the Declaration of Helsinki.

\section{Management before the catheter ablation}

Electrocardiography and a chest X-ray were performed within 3 months before the catheter ablation. A transthoracic echocardiogram was performed to evaluate the left atrial diameter and left ventricular ejection fraction. All patients underwent multi-detector row computed tomography (CT) within 1 week before the catheter ablation to obtain the configuration of the left atrial cavity and rule out any thrombi in the left atrial appendage. The slice data of the image was reconstructed into a 3-dimensional volume rendering by computer software (ZAIO station2, ZAIOSOFT, Tokyo, Japan). The 3-dimensional image clarified the anatomy of the pulmonary veins consisting of the left superior pulmonary vein (LSPV), left inferior pulmonary vein (LIPV), RSPV, and right inferior pulmonary vein (RIPV). The isolated right middle lobe branch was regarded as the right middle pulmonary vein (RMPV). The maximal diameter and ostial area of the right sided pulmonary veins were measured. Each PV ostial area was measured at the level of the intervenous carina by manually tracing the contour.

All patients received anticoagulation therapy for at least 3 weeks before the ablation procedure. Antiarrhythmic drugs were discontinued for more than 5 half-lives before the ablation procedure, and amiodarone was not administered before the ablation procedure in all patients.

\section{Catheter ablation procedure}

Catheter ablation was performed under general anesthesia using propofol and dexmedetomidine. A single-use supraglottic airway device (i-gel, NIHON KOHDEN, Tokyo, Japan) was inserted in all study patients, and an esophageal temperature thermocouple catheter (Esophaster, Japan Lifeline, Tokyo, Japan) was inserted through this airway device into the esophagus for continuous monitoring. Vascular access was acquired through the right internal jugular and femoral veins. A 20-pole catheter (BeeAT, Japan Lifeline, Tokyo, Japan) was inserted into the coronary sinus (CS) for electrogram recording, atrial pacing, and defibrillation. For the index ablation procedure, 2 transseptal punctures were performed: one for the 12-Fr inner-diameter deflectable sheath (CardioFocus, MA, USA) and VGLA catheter and the other for a circular mapping catheter. Heparin was given as intravenous boluses followed by a constant infusion to maintain an activated clotting time of $>300$ seconds during the ablation procedure. An EnSite NavX navigation system (St. Jude Medical, St. Paul, MN, USA) was used for 3-dimensional mapping.

A mapping catheter (EPstar libero, Japan Lifeline, Tokyo, Japan) was used to construct the geometry of the left atrium (LA) and pulmonary veins (PVs). The bipolar electrogram filters were set between 30 and 500 Hz. The baseline PV electrograms were recorded using fluoroscopy via the circular mapping catheter that was positioned at the PV ostium. The first-generation VGLA catheter (HeartLight, CardioFocus, MA, USA) was inserted into the LA, and expanded to occlude each PV. The compliant balloon was inflated to multiple pressures to change its size so that the balloon/tissue contact was maximized regardless of the size or shape of 
the target PV ostium. The laser energy level was titrated according to the degree of tissue exposure between 5.5 and $12 \mathrm{~W}$ using LightTrack ${ }^{\mathrm{TM}}$ software (CardioFocus, MA, USA). Every energy application was applied for 20 to 30 seconds. To avoid any laser balloon (LB) rupture, a dose of $5.5 \mathrm{~W}$ for 30 seconds was applied in areas that were adjacent to blood. By using the adjustable $30^{\circ}$ aiming arc for guidance, the laser energy (5.5-16 W, 20-30 seconds) was delivered around the PV ostium in a contiguous manner with a $50 \%$ lesion overlap. Ablation was prematurely terminated when the esophageal temperature reached 39. The VGLA was performed in the order of the LSPV, LIPV, RSPV, and RIPV. The RMPV was simultaneously isolated while an encircling ablation was performed for the RSPV or RIPV with the VGLA. After the initial encirclement, the PVI status was again assessed, and additional laser energy was delivered at electrical gap sites in cases of an unsuccessful PVI. If the PVI could not be successfully accomplished only with the LB applications, an irrigated radiofrequency (RF) catheter (TactiCath, Abbott, Illinois, USA) was used for a touch-up ablation to eliminate all gap conduction sites. When PV electrograms were present, isolated PV potentials identified exit block. A successful PVI was defined as bidirectional block between the left atrium (LA) and inside the circumferential PVI area, which was confirmed by the electrical dissociation between the LA and PV during pacing with the electrode catheter positioned inside the PVI area and electrode catheter positioned inside the CS. A cavotricuspid isthmus ablation was also performed when atrial flutter was observed during the ablation procedure or on the discretion of the operator. A non-PV focus ablation was not performed. The radiofrequency energy was set at the maximum output of $35 \mathrm{~W}$, and the catheter tip temperature was not allowed to exceed $42 \mathrm{degC}$.

During ablation of the RSPV or RIPV, continuous and stable right phrenic nerve pacing was performed and the CMAPs were continuously recorded during the ablation procedure. A 10-pole catheter was placed in the right subclavian vein or superior vena cava to pace the right phrenic nerve. The threshold for capturing the diaphragm was measured, and pacing was performed at a rate of 40 pulses per minute at an output exceeding the pacing threshold by $10 \%$ as previously described $_{8 \text { ) }}$. We defined PNI group as the group in which the CMAPs decreased by more than $30 \%$ during the LB application. After the successful PVI was achieved, it was again reassessed after a 15-minute post-ablation waiting period.

During the ablation of the RSPV, the position of the LB was confirmed under fluoroscopy (Infinix, CANNON, Tokyo, Japan). The fluoroscopic images were stored and analyzed with Goodnet software (GOODMAN, Nagoya, Japan). The distance between the LB apex and cardiac shadow (Figure.1 ) was measured in the anteroposterior position.

\section{Follow-up}

All patients were routinely seen at 1 and 3 months and every 3 months after discharge in the outpatient clinic. At those follow-up visits, a physical examination, ECG recording, and review of the patient's symptoms were performed. All patients were instructed to tell the attending physician if there were any symptoms suggestive of an arrhythmia recurrence. In that case, the rhythm was assessed using a 12-lead ECG. A Holter ECG examination was performed at 3 and 12 months after the VGLA procedure. An atrial tachyarrhythmia recurrence was defined as any sustained $\mathrm{AF} / \mathrm{AT}$ lasting for $>30$ seconds, which appeared $>3$ months after the blanking period of the catheter ablation. Clinical success evaluated at 12 months after the initial PVI procedure was defined as the freedom from any symptomatic PAF assessed by a clinical assessment, 12-lead ECG, and Holter ECG recordings.

A chest X-ray examination was also undertaken at 1 and 3 months after discharge. Persistent PNI was defined as an elevated right-sided diaphragm noted on post-procedural chest X-ray obtained on the following day of the VGLA, which persisted even after the VGLA procedure. Patients with persistent PNI had a chest $\mathrm{X}$-ray examination taken at each outpatient clinic visit and were evaluated until the resolution of the PNI.

\section{Statistical analysis}

Continuous variables are expressed as the mean $\pm \mathrm{SD}$, and significant differences were analyzed using a Student's t test. Categorical data are expressed as the number and percentages, and were compared using an $\chi^{2}$ test or Fisher's exact test. Univariate and multivariate Logistic analyses were performed on candidate 
variables to predict the dichotomous outcome. A logistic regression analysis was applied to candidate variables to predict the dichotomous outcome of PNI. The appropriate cutoff value was determined as the sum of the highest sensitivity and specificity using a receiver-operating characteristic (ROC) curve. A 95\% CI was shown as the area under the curve (AUC). A probability value of $<0.05$ was deemed significant. Statistical analyses were conducted using the EZR software (Saitama Medical Center, Jichi Medical University, Saitama, Japan), which is a graphical user interface for R (The R Foundation for Statistical Computing, Vienna, Austria).

\section{Results}

\section{Baseline characteristics}

Table 1 shows the baseline clinical characteristics of the study patients. A total of 130 patients $(67 \%$ male, age $66 \pm 12$ years) who underwent a VGLA as an initial PVI were analyzed in the study. The mean $\mathrm{CHA}_{2} \mathrm{DS}_{2}$-VASc score was $2.0 \pm 1.5$ and mean left atrial diameter (LAD) $38.4 \pm 6.1 \mathrm{~mm}$. The RIPV could not be isolated in 4 patients, because the VGLA was prematurely terminated due to the sudden failure to capture the phrenic nerve, which lasted until the end of the ablation procedure. Except for those 4 patients, all four PVs were successfully isolated by the VGLA. An LB rupture was provoked in 3 patients, and 26 patients needed touch up RF ablation. There were no significant differences in the age, gender, body mass index (BMI), or comorbidities such as hypertension, diabetes, and a history of congestive heart failure or a stroke between those with and without PNI during the VGLA procedure. The LA diameter and left ventricular ejection fraction (LVEF) were also comparable between the two groups. Moreover, there was no significant difference in the total procedure time, energy needed to isolate each PV or the distance between the cardiac shadow and LB apex, between the two groups (Table 1 ).

\section{Phrenic nerve injury during the VGLA}

PNI developed in a total of 20 out of 130 (15.4\%) patients during the VGLA. The PNI occurred during the RSPV and RIPV isolation in 17 and 3 patients, respectively. Table 2 summarizes the details of the patients who developed PNI during the procedure. The mean time from the initiation of the laser application to the development of PNI was $3.6 \pm 1.0$ (ranging from 1.5 to 4.4 ) seconds, and the mean energy was 31.8 \pm 10.6 (ranging from 12.8 to 52.8) J. Figure 2 shows an example of the development of PNI during the VGLA. Both the stable phrenic nerve stimulation and CMAP amplitude recordings were confirmed before laser energy application to the RSPV. However, the loss of capture of the phrenic nerve during electrical stimulation occurred all of sudden within only 1.5 seconds. The sites where the PNI was provoked were as follows: carina of the RSPV in 8, anterior RSPV in 7, anterior RIPV in 3, and roof of the RSPV in 2 patients, respectively (Figure 3 ).

The optimal cut-off value of the RSPV diameter and RSPV ostial area (RSPVOA) for predicting PNI were investigated. The area under the ROC curve was used to assess the predictive power of the RSPV diameter and RSPVOA for PNI. The optimal cutoff values for the RSPV diameter and RSPVOA were $19.0 \mathrm{~mm}$ and $238.0 \mathrm{~mm}^{2}$, respectively. The AUC of the RSPV diameter and RSPVOA were $0.72(95 \%$ confidence interval [CI] 0.61-0.83, sensitivity $95.0 \%$, specificity $37.3 \%)$ and 0.79 (95\% confidence interval [CI] 0.69-0.90, sensitivity $95.0 \%$, specificity $58.2 \%$ ), respectively.

\section{Predictors of phrenic nerve injury during the VGLA}

A Multivariate analysis showed that a large RSPVOA was an independent predictor of PNI (hazards ratio $[\mathrm{HR}]=1.02,95 \%$ confidence interval $[\mathrm{CI}] 1.01-1.03, \mathrm{p}<0.01)$, and a large balloon size during the RSPV ablation was an independent risk reduction factor for PNI (hazards ratio $[\mathrm{HR}]=0.71,95 \%$ confidence interval $[\mathrm{CI}]$ 0.54-0.93, $\mathrm{p}=0.03$ ) (Table 3 ). The distance between the cardiac shadow and LB apex was not significantly associated with the occurrence of PNI.

\section{Follow-up}

After a mean follow-up of $331 \pm 121$ (ranging from 90 to 572) days, atrial tachyarrhythmias recurred in 
20 patients $(15.4 \%)$. Persistent PNI was provoked in 13 patients during the VGLA, and it persisted even after discharge. However, complete recovery from the PNI was observed in 12 patients during the follow-up period.

\section{Discussion}

In the present retrospective single-center study, 130 patients who underwent VGLA using the first generation LB were analyzed. The main findings of the present study were as follows: (1) the time from the initiation of the laser application to the occurrence of PNI was very short, (2) a large RSPV and small LB size were predictors of PNI, and (3) the PNI during the LB ablation was reversible in the majority of patients.

\section{The characteristics of PNI during VGLA}

The continuous monitoring of the CMAP during the PVI is a useful technique for early detection of $\mathrm{PNI}_{9,10)}$. Moreover, a reduction in the amplitude of the CMAP by more than $30 \%$ during the VGLA has been reported to be at risk for developing phrenic nerve palsy ${ }_{11,12}$. In our study, the reduction in the CMAP amplitude occurred in a very rapid fashion during the VGLA, and the mean time to develop PNI was only $3.6 \pm 1.0$ seconds. The time to the PNI occurrence was very short during the VGLA, therefore, continuous CMAP monitoring cannot be regarded as a useful tool for preventing PNI during VGLA. The present study showed that 17 patients developed PNI during an RSPV ablation and 3 during an RIPV ablation. This result was compatible with that of the PVI using the $\mathrm{CB}$, and which is considered to be associated with the anatomical course of the phrenic nerve 13$)$.

Previous studies have demonstrated that laser energy can create deeper and more discrete lesions in the left atrial antrum-pulmonary vein region than radiofrequency ablation 14 ). Moreover, laser energy is absorbed by the water in the cardiac tissue, and the maximal heat initially reaches beyond the endocardial level ${ }_{15}$, which may cause PNI quickly.

Tohoku et al. reported the majority of the PNIs during CB ablation are transient whereas the majority of PNI during VGLA are persistent ${ }_{16}$. They discussed that the difference in the temperature between the blood and heated tissue of the phrenic nerve is smaller than that of the CB, which makes it difficult to cool heated tissue in the phrenic nerve, and persistent PNI occurs frequently in VGLA 16 ).

\section{Predictors of phrenic nerve injury during VGLA}

Nagase et al. and Okishige et al. reported that the lesion depth, lesion volume, and maximum lesion diameter increased according to not only the laser output but also the delivered total laser energy in an in vitro model ${ }_{17,18)}$. However, our study showed there was no significant difference in the mean output and delivered total energy between the groups with and without PNI. Moreover, the distance between the LB and cardiac shadow on fluoroscopic image had no significant relationship with the incidence of PNI.

Because the CMAP was not useful for preventing PNI due to the extremely rapid reduction in the CMAP amplitude during the VGLA, the detection of other predictors of PNI are so important. The present study showed that a larger ostium of the RSPV and smaller LB size were predictors of PNI. Therefore, the maneuver to make the LB size the largest relative to the RSPVOA turned out to be the most effective strategy to prevent PNI, which encourages us to ablate the RSPV with a large sized LB as much as possible. Recently, contrast $\mathrm{CT}$ has been performed before ablation in order to construct the geometry of the heart chambers merged with a 3-dimensional mapping system or to confirm a thrombus in the LA. We must pay attention to the PNI in patients with a large RSPV when a relatively large RSPV is recognized by CT images. Sano et al. reported that pacing at each PV ostium before the CB ablation could be effective in predicting $\mathrm{PNI}_{19}$ ). Therefore, when VGLA will be performed in patients with a relatively large RSPVOA, pacing inside the PVs might be recommended to avoid the PNI.

\section{Comparison to the previous reports}

In our study, persistent PNI occurred in 13 patients (10.0\%), which persisted even after discharge. Previous studies on VGLA reported that the incidence of PNI was $0-8.6 \%_{2,3,20-24)}$. Meta-analysis reported PNI was 
the most common procedural complication following the VGLA, which was observed in 32 of $1162(2.6 \%)$ procedures across 16 studies $_{25}$ ). The incidence of PNI was higher in the present study than prior studies. This may be explained by the following reasons. First, the LB size in the present study might have been relatively smaller than that in the other previous reports. The present study demonstrated that the LB size was an independent risk reduction factor for provoking PNI. A larger LB size was accordingly associated with a lower incidence of the PNI (hazards ratio $[\mathrm{HR}]=0.71,95 \%$ confidence interval $[\mathrm{CI}]$ 0.54-0.93, $\mathrm{p}=$ 0.03). To the best to our knowledge, no previous study has reported the relationship between the LB size and incidence of PNI. Second, significant differences existed with regard to the definition of persistent PNI. We defined that persistent PNI was provoked even when only a subtle difference in the diaphragm was recognized in the post-procedural chest X-ray compared to the pre-procedural chest X-ray. None of the patients with persistent PNI underwent spirometry or a sniffing test, and therefore, we failed to systematically assess the study patients in terms of investigating the genuine incidence of persistent PNI. However, the PNI provoked by the VGLA in the present study was reversible in the majority of the patients during the follow-up period. The final incidence of persistent PNI was almost comparable to that of the recently published studies on VGLA $_{2,3,20-24)}$.

\section{Study limitations}

Our study had some limitations. First, this study was a single-center, retrospective analysis and the study sample size was relatively small. A larger volume study is needed to verify our results. Second, the pacing cycle length was longer than in the other study ${ }_{11}$. Franceschi $\mathrm{F}$ et al. reported that a $30 \%$ reduction in the diaphragmatic CMAP amplitude was a risk factor for provoking PNI for the first time ${ }_{11)}$. They performed pacing of the right-sided PN at a frequency of 60 pulses per minute. We might have been able to reduce the incidence of PNI, which occurred within a few seconds, in most cases, if we had paced the PN with a higher pacing rate than in the present study.

\section{Conclusions}

The incidence of PNI during the VGLA procedure was relatively high in the present study, but the PNI improved in most of the cases. A larger ostium of the RSPV and smaller LB size were reliable predictors of PNI. A strategy to avoid PNI might be to make effort to attempt to expand the LB as large as possible relative to the size of the RSPV ostium.

\section{References}

1. Calkins H, Hindricks G, Cappato R, et al. 2017 HRS/EHRA/ECAS/APHRS/SOLAECE expert consensus statement on catheter and surgical ablation of atrial fibrillation. Heart Rhythm 2017;14:e275e444.

2. Dukkipati SR, Cuoco F, Kutinsky I, Aryana A, Bahnson TD, Lakkireddy D, Woollett I, Issa ZF, Natale A, Reddy VY; HeartLight Study Investigators. Pulmonary vein isolation using the visually guided laser balloon: a prospective, multicenter, and randomized comparison to standard radiofrequency ablation. J Am Coll Cardiol 2015;66:1350-1360.

3. Schmidt B, Neuzil P, Luik A, Osca Asensi J, Schrickel JW, Deneke T, Bordignon S, Petru J, Merkel M, Sediva L, Klostermann A, Perrotta L, Cano O, Chun KRJ. Laser Balloon or Wide-Area Circumferential Irrigated Radiofrequency Ablation for Persistent Atrial Fibrillation. Circ Arrhthm Electrophysiol 2017;10:e005767

4. Wei Y, Zhang N, Jin Q, Pan W, Xie Y, Chen K, Ling T, Lin C, Bao Y, Luo Q, Xing C, Wu L. Comparison of efficacy and safety of laser balloon and cryoballoon ablation for atrial fibrillation - a meta-analysis. J Interv Card Electrophysiol 2019;54:237-245.

5. Okumura Y, Henz BD, Bunch TJ, Dalegrave C, Johnson SB, Packer DL. Distortion of right superior pulmonary vein anatomy by balloon catheters as a contributor to phrenic nerve injury. J Cardiovasc Electrophysiol 2009;20:1151-1157. 
6. Saitoh Y, Ströker E, Irfan G, Mugnai G, Ciconte G, Hünük B, Velagić V, Overeinder I, Tanaka K, Brugada P, de Asmundis C, Chierchia GB; Fluoroscopic position of the second-generation cryoballoon during ablation in the right superior pulmonary vein as a predictor of phrenic nerve injury. Europace 2016;186:1179-1186.

7. Nagase T, Asano S, Goto K, Ikeda Y, Kato R, Matsumoto K. Phrenic nerve injury contemporaneous with acute decrease in compound motor action potential during laser balloon pulmonary vein isolation for atrial fibrillation. Heart Rhythm Case Reports 2019;5:83-87.

8. Okishige K, Aoyagi H, Kawaguchi N, Katoh N, Yamashita M, Nakamura T, Kurabayashi M, Suzuki H, Asano M, Gotoh K, Shimura T, Yamauchi Y, Kanazawa T, Sasano T, Hirao K. Novel method for earlier detection of phrenic nerve injury during cryoballoon applications for electrical isolation of pulmonary veins in patients with atrial fibrillation. Heart Rhythm 2016;13:1810-1816.

9. Franceschi F, Dubuc M, Guerra PG, Khairy P. Phrenic nerve monitoring with diaphragmatic electromyography during cryoballoon ablation for atrial fibrillation: the first human application. Heart Rhythm 2011;8:1068-1071.

10. Miyazaki S, Hachiya H, Taniguchi H, Nakamura H, Ichihara N, Usui E, Kuroi A, Takagi T, Iwasawa J, Hirao K, Iesaka Y. Prospective evaluation of bilateral diaphragmatic electromyograms during cryoballoon ablation of atrial fibrillation. J Cardiovasc Electrophysiol 2015;26:622-628.

11. Franceschi F, Koutbi L, Mancini J, Attarian S, Prevôt S, Deharo JC. Novel electromyographic monitoring technique for prevention of right phrenic nerve palsy during cryoballoon ablation. Circ Arrhythm Electrophysiol 2013;6:1109-1114.

12. Mondesert B, Andrade JG, Khairy P, Guerra PG, Dyrda K, Macle L, Rivard L, Thibault B, Talajic M, Roy D, Dubuc M, Shohoudi A. Clinical experience with a novel electromyographic approach to preventing phrenic nerve injury during cryoballoon ablation in atrial fibrillation. Circ Arrhythm Electrophysiol 2014;7:605-611.

13. Okumura Y, Henz BD, Bunch TJ, Dalegrave C, Johnson SB, Packer DL. Distortion of Right Superior Pulmonary Vein Anatomy by Balloon Catheters as a Contributor to Phrenic Nerve Injury. J Cardiovasc Electrophysiol 2009;20:1151-1157.

14. Figueras I, Ventura RM, Mărgulescu AD, Benito EM, Alarcón F, Enomoto N, Prat-Gonzalez S, Perea RJ, Borràs R, Chipa F, Arbelo E, Tolosana JM, Brugada J, Berruezo A, Mont L. Postprocedural LGE-CMR comparison of laser and radiofrequency ablation lesions after pulmonary vein isolation. J Cardiovasc Electrophysiol 2018;29:1065-1072.

15. Ücer E, Janeczko Y, Seegers J, Fredersdorf S, Friemel S, Poschenrieder F, Maier LS, Jungbauer CG. A RAndomized Trial to compare the acute reconnection after pulmonary vein Isolation with LaserBalloON versus radiofrequency Ablation: RATISBONA trial. J Cardiovasc Electrophysiol 2018;29:733739 .

16. Tohoku S, Chen S, Last J, Bordignon S, Bologna F, Trolese L, Zanchi S, Bianchini L, Schmidt B, Chun KRJ. Phrenic nerve injury in atrial fibrillation ablation using balloon catheters: Incidence, characteristics and clinical recovery course. J Cardiovasc Electrophysiol 2020 May 17. doi: 10.1111/jce.14567.

17. Nagase T, Asano S, Yukino M, Mori H, Goto K, Ikeda Y, Iwanaga S, Muramatsu T, Mukaida H, Kato R, Matsumoto K. Influence of various energy settings and overlap ratios on size and continuity of lesions in a laser balloon ablation in vitro model. J Cardiovasc Electrophysiol 2019;30:1330-1338.

18. Okishige K, Shigeta T, Nakamura RA, Hirao T, Yoshida H, Oda A, Yamauchi Y, Sasano T, Hirao K. Experimental Study With Regard to the Effects of Energy Titration of the Laserballoon on the Lesion Creation Using Porcine Myocardium. Int Heart J 2020;61:121-127. 
19. Sano M, Urushida T, Matsukura G, Sakakibara T, Suwa K, Naruse Y, Ohtani H, Saotome M, Maekawa Y. Clinical feasibility of pulmonary vein orifice pacing for the prediction of phrenic nerve injury during cryoballoon ablation of atrial fibrillation. J Cardiovasc Electrophysiol 2019;30:1200-1206.

20. Stockigt F, Kohlmann AT, Linhart M, Nickenig G, Andrie RP, Beiert T, Schrickel JW. Laserballoon and Cryoballoon Pulmonary Vein Isolation in Persistent and Longstanding Persistent Atrial Fibrillation. Pacing Clin Electrophysiol 2016;39:1099-1107.

21. Sediva L, Petru J, Skoda J, Janotka M, Chovanec M, Reddy V, Neuzil P. Visually guided laser ablation: a single-centre long-term experience. Europace 2014;16:1746-1751.

22. Perrotta L, Bordignon S, Dugo D, Furnkranz A, Chun KJ, Schmidt B: How to learn pulmonary vein isolation with a novel ablation device: learning curve effects using the endoscopic ablation system. J Cardiovasc Electrophysiol 2014;25:1293-1298.

23. Metzner A, Wissner E, Schmidt B, Chun J, Hindricks G, Piorkowski C, Ouyang F, Kuck KH. Acute and long-term clinical outcome after endoscopic pulmonary vein isolation: results from the first prospective, multicenter study. J Cardiovasc Electrophysiol 2013;24:7-13.

24. Bordignon S, Boehmer MC, Klostermann A, Fuernkranz A, Perrotta L, Dugo D, Chun KR, Schmidt B. Visually guided pulmonary vein isolation in patients with persistent atrial fibrillation. Europace 2016;18:538-542.

25. Reynolds MR, Zheng Q, Doros G. Laser Balloon Ablation for AF: A Systematic Review and MetaAnalysis. J Cardiovasc Electrophysiol 2018;29:1363-1370.

26.

Table 1 . Baseline characteristics and procedural characteristics of the patients with and without PNI

\begin{tabular}{|c|c|c|c|}
\hline Variable & Overall $(\mathrm{n}=130)$ & PNI - $(n=110)$ & $\mathrm{PNI}+(\mathrm{n}=20)$ \\
\hline Age, years & $66.0 \pm 11.8$ & $65.8 \pm 11.8$ & $67.2 \pm 12.3$ \\
\hline Female, $\mathrm{n}$ & $43(33.1 \%)$ & $33(30.0 \%)$ & $10(50.0 \%)$ \\
\hline BMI, $\mathrm{kg} / \mathrm{m}^{2}$ & $23.8 \pm 3.7$ & $24.1 \pm 3.7$ & $22.6 \pm 3.6$ \\
\hline Hypertension, $\mathrm{n}$ & $54(41.5 \%)$ & $44(40.0 \%)$ & $10(50.0 \%)$ \\
\hline Diabetes, $\mathrm{n}$ & $10(7.7 \%)$ & $8(7.3 \%)$ & $2(10.0 \%)$ \\
\hline Heart failure, $\mathrm{n}$ & $8(6.2 \%)$ & $5(4.5 \%)$ & $3(15.0 \%)$ \\
\hline Stroke or TIA, $\mathrm{n}$ & $11(8.5 \%)$ & $11(10.0 \%)$ & $0(0 \%)$ \\
\hline $\mathrm{CHA}_{2} \mathrm{DS}_{2}$ VASc score & $2.0 \pm 1.5$ & $2.0 \pm 1.5$ & $2.3 \pm 1.3$ \\
\hline LA diameter, mm & $38.4 \pm 6.1$ & $38.8 \pm 6.1$ & $36.1 \pm 5.6$ \\
\hline LVEF, \% & $65.9 \pm 9.0$ & $65.9 \pm 8.4$ & $65.7 \pm 12.0$ \\
\hline CT parameters & CT parameters & CT parameters & CT parameters \\
\hline RSPV diameter, mm & $19.7 \pm 2.8$ & $19.4 \pm 2.6$ & $21.9 \pm 2.9$ \\
\hline RSPVOA, $\mathrm{mm}^{2}$ & $241.1 \pm 49.9$ & $233.1 \pm 46.4$ & $284.7 \pm 47.0$ \\
\hline RIPV diameter, mm & $18.9 \pm 2.9$ & $18.9 \pm 2.9$ & $18.5 \pm 3.0$ \\
\hline RIPVOA, $\mathrm{mm}^{2}$ & $230.6 \pm 63.7$ & $231.8 \pm 64.2$ & $224.4 \pm 62.4$ \\
\hline Total procedure time, min & $172.7 \pm 31.7$ & $173.9 \pm 32.4$ & $165.7 \pm 27.0$ \\
\hline Ablation time, $\min$ & $84.8 \pm 32.5$ & $86.2 \pm 34.3$ & $76.4 \pm 18.6$ \\
\hline Fluoroscopy time, min & $55.5 \pm 15.3$ & $55.8 \pm 15.5$ & $54.0 \pm 14.4$ \\
\hline Laser Mean output, W & Laser Mean output, W & Laser Mean output, W & Laser Mean output, \\
\hline RSPV & $8.7 \pm 1.4$ & $8.7 \pm 1.5$ & $8.7 \pm 1.3$ \\
\hline RIPV & $7.9 \pm 1.4$ & $7.9 \pm 1.4$ & $7.7 \pm 1.6$ \\
\hline LSPV & $8.6 \pm 1.5$ & $8.6 \pm 1.5$ & $8.6 \pm 1.5$ \\
\hline LIPV & $7.8 \pm 1.4$ & $7.7 \pm 1.4$ & $7.9 \pm 1.6$ \\
\hline Total energy, J & Total energy, J & Total energy, J & Total energy, J \\
\hline
\end{tabular}




\begin{tabular}{llll}
\hline Variable & Overall $(\mathrm{n}=130)$ & PNI $-(\mathrm{n}=110)$ & PNI $+(\mathrm{n}=20)$ \\
\hline RSPV & $6014.9 \pm 2667.3$ & $6164.2 \pm 2785.2$ & $5248.1 \pm 1752.6$ \\
RIPV & $4489.5 \pm 1506.7$ & $4481.1 \pm 1436.9$ & $4528.0 \pm 2000.5$ \\
LSPV & $6768.2 \pm 3128.5$ & $6706.1 \pm 3000.5$ & $7106.6 \pm 3823.8$ \\
LIPV & $4628.3 \pm 2359.4$ & $4501.0 \pm 2213.9$ & $5304.9 \pm 2999.7$ \\
Dormant conduction, $\mathrm{n}$ & $19(14.6 \%)$ & $15(13.7 \%)$ & $4(20 \%)$ \\
Radiofrequency touch up, $\mathrm{n}$ & $26(20.0 \%)$ & $21(19.1 \%)$ & $5(25.0 \%)$ \\
Distance between cardiac shadow and LB apex & $22.2 \pm 7.0$ & $22.2 \pm 7.1$ & $22.5 \pm 6.5$ \\
CTI ablation, n & $119(91.5 \%)$ & $101(91.8 \%)$ & $18(90.0 \%)$ \\
\hline
\end{tabular}

$\mathrm{PNI}=$ phrenic nerve injury; $\mathrm{BMI}=$ body mass index; TIA $=$ transient ischemic attack; LA = left atrium; $\mathrm{LVEF}=$ left ventricular ejection fraction; RSPV = right superior pulmonary vein; RSPVOA = right superior pulmonary vein ostial area; RIPV = right inferior pulmonary vein; RIPVOA = right inferior pulmonary vein ostium area; LSPV = left superior pulmonary vein; LIPV = left inferior pulmonary vein; LB = laser balloon.

Values are expressed as the mean $\pm \mathrm{SD}$ or as $\mathrm{n}(\%)$. ${ }^{*} \mathrm{P}$ value is compared between the PNI - and PNI + groups, respectively.

Table 2 . Details of the patients who developed PNI during the procedure.

\begin{tabular}{|c|c|c|c|c|c|c|}
\hline Case Number & PV with PNI & PV with PNI & Laser Output (W) & Time to PNI (sec) & Energy to PNI $(\mathrm{J})$ & Amplitude c \\
\hline & RSPV & RIPV & & & & Maximum ( \\
\hline Patient 1 & + & - & 8.5 & 4.4 & 37.4 & 0.74 \\
\hline Patient 2 & + & - & 8.5 & 4.4 & 37.4 & 0.82 \\
\hline Patient 3 & + & - & 8.5 & 2.9 & 24.7 & 0.81 \\
\hline Patient 4 & + & - & 8.5 & 4.4 & 37.4 & 0.42 \\
\hline Patient 5 & + & - & 8.5 & 2.9 & 24.7 & 0.47 \\
\hline Patient 6 & + & - & 8.5 & 2.9 & 24.7 & 0.94 \\
\hline Patient 7 & + & - & 8.5 & 4.4 & 24.2 & 0.46 \\
\hline Patient 8 & + & - & 8.5 & 4.4 & 37.4 & 0.66 \\
\hline Patient 9 & + & - & 8.5 & 1.5 & 12.8 & 0.64 \\
\hline Patient 10 & - & + & 8.5 & 2.9 & 24.7 & 1.0 \\
\hline Patient 11 & + & - & 10 & 4.4 & 44 & 0.39 \\
\hline Patient 12 & + & - & 10 & 2.9 & 29 & 0.34 \\
\hline Patient 13 & + & - & 12 & 1.5 & 18 & 0.70 \\
\hline Patient 14 & + & - & 10 & 4.4 & 44 & 0.46 \\
\hline Patient 15 & - & + & 10 & 4.4 & 44 & 0.66 \\
\hline Patient 16 & + & - & 8.5 & 4.4 & 37.4 & 0.54 \\
\hline Patient 17 & + & - & 12 & 2.9 & 34.8 & 0.46 \\
\hline Patient 18 & - & + & 5.5 & 2.9 & 16 & 0.72 \\
\hline Patient 19 & + & - & 7 & 4.4 & 30.8 & 0.33 \\
\hline \multirow[t]{2}{*}{ Patient 20} & + & - & 12 & 4.4 & 52.8 & 0.51 \\
\hline & $17(85.0 \%)$ & $3(15.0 \%)$ & $9.0 \pm 1.8$ & $3.6 \pm 1.0$ & $31.8 \pm 10.6$ & \\
\hline
\end{tabular}

$\mathrm{CMAP}=$ compound motor action potential; $\mathrm{PNI}=$ phrenic nerve injury; RSPV $=$ right superior pulmonary vein; RIPV = right inferior pulmonary vein

Table 3 . Univariate and multivariable analyses for the prediction of PNI 


\begin{tabular}{llllc}
\hline & Univariate analysis & Univariate analysis & Multivariate analysis & Multiv \\
\hline & HR $(95 \% \mathrm{CI})$ & P value & HR $(95 \%$ CI $)$ & P valu \\
Age & $1.01(0.97-1.05)$ & 0.61 & & \\
Female & $2.33(0.88-6.14)$ & 0.09 & & \\
BMI & $0.88(0.75-1.03)$ & 0.10 & & \\
$\mathrm{CHA}_{2} \mathrm{DS}_{2}$ VASc score & $1.13(0.82-1.56)$ & 0.47 & & \\
LA diameter & $0.92(0.85-1.01)$ & 0.07 & $1.02(1.01-1.03)$ & 0.01 \\
RSPVOA & $1.02(1.01-1.03)$ & $<0.01$ & $0.72(0.53-0.98)$ & 0.03 \\
LB size in RSPV & $0.71(0.54-0.93)$ & 0.01 & & \\
Distance between cardiac shadow and LB apex & $1.01(0.94-1.08)$ & 0.89 & & \\
\hline
\end{tabular}

$\mathrm{PNI}=$ phrenic nerve injury; $\mathrm{BMI}=$ body mass index; $\mathrm{LA}=$ left atrium; RSPV $=$ right superior pulmonary vein; $\mathrm{RSPVOA}=$ right superior pulmonary vein ostial area; $\mathrm{LB}=$ laser balloon; $\mathrm{HR}=$ hazard ratio; $\mathrm{CI}=$ confidence interval.

\section{Figure Legends}

Figure 1 .

Positioning of the LB in relation to the cardiac shadow under fluoroscopic guidance in the anteroposterior projection during the RSPV ablation. The distance between the cardiac shadow and LB apex was measured.

\section{Figure 2 .}

An example of provoking PNI during VGLA. Phrenic nerve pacing was performed at a rate of 40 pulses per minute at an output $7 \mathrm{~V}$. The amplitude of the CMAP was stably recorded as $0.65-0.70 \mathrm{mV}$. However, pacing failure of the phrenic nerve occurred within only 1.5 seconds just after the initiation of the laser application.

\section{Figure 3.}

The distribution of the PNI sites. Eight were located at the carina of the RSPV, 7 at the anterior RSPV, 3 at the anterior RIPV, and 2 on the roof of the RSPV, respectively.

$\mathrm{PNI}=$ phrenic nerve injury; RSPV $=$ right superior pulmonary vein; RSPV $=$ right inferior pulmonary vein.

\section{Figures}

\section{Figure 1 .}




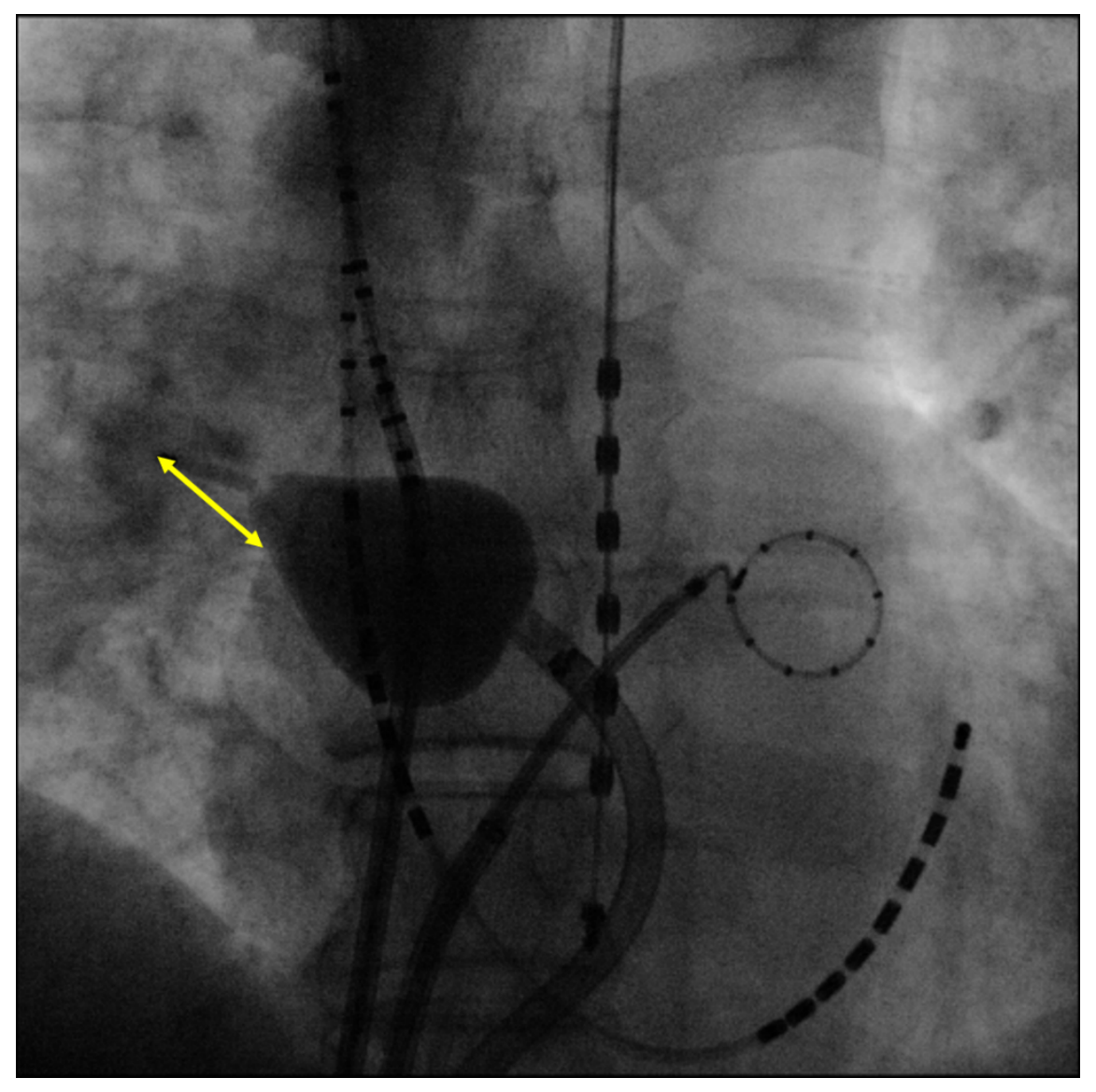

Figure 2 .

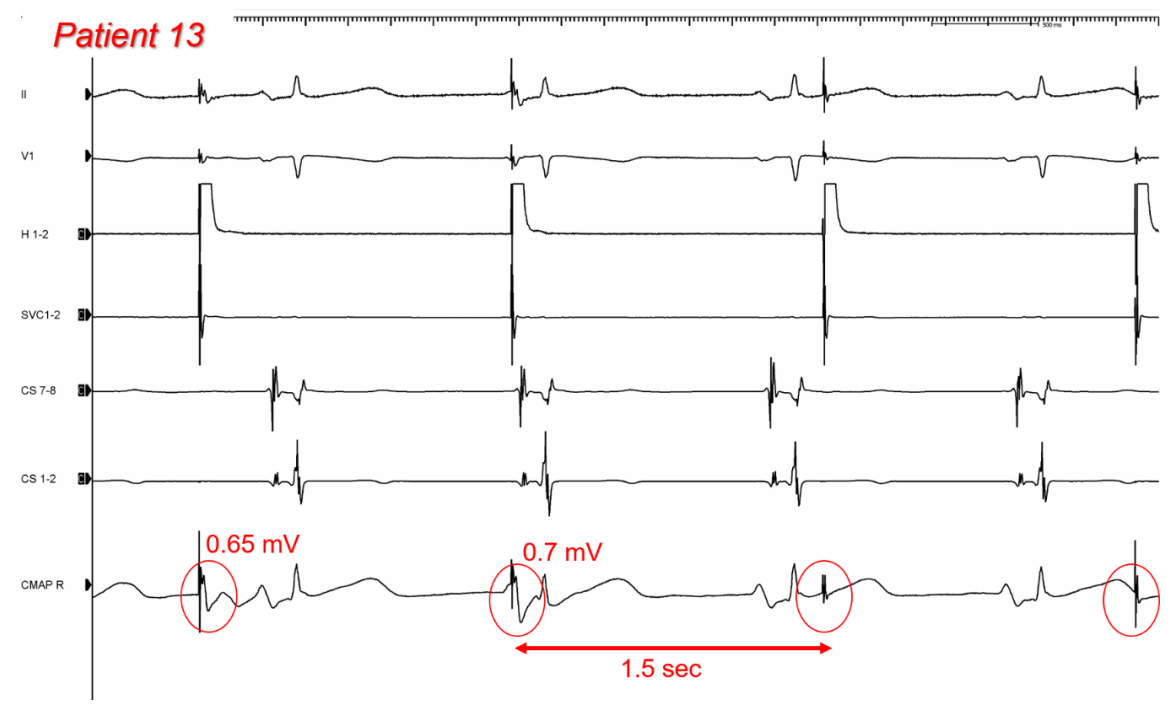

Figure 3. 


\section{Distribution of sites of the PNI}
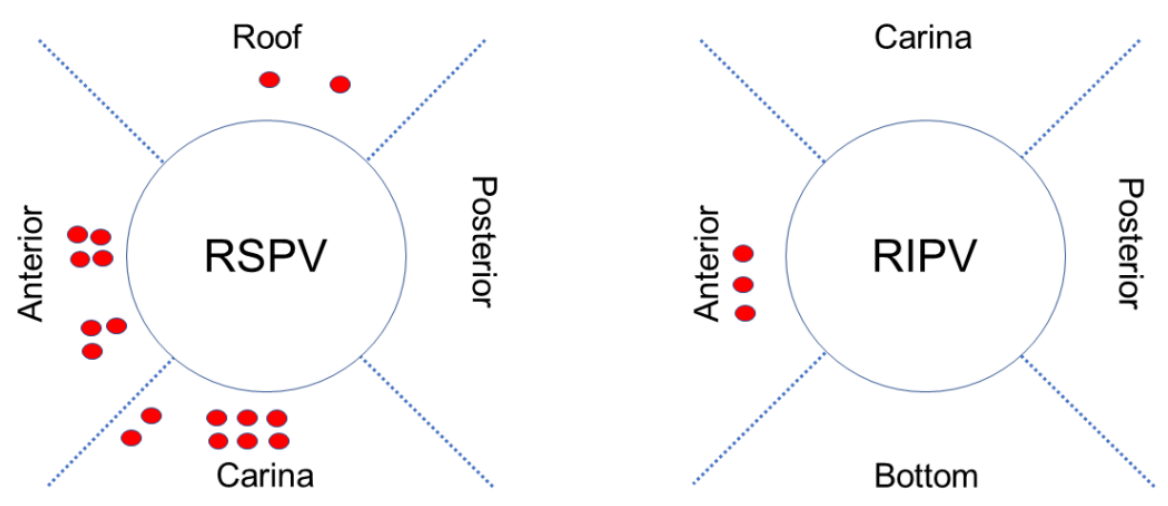